\title{
EFFECTS OF INTRAOPERATIVE LIQUID AND RESTRICTIVE BLOOD TRANSFUSION MANAGEMENT TO HOSPTIAL DISCHARGE IN HIGH RISK PATIENTS ON MAJOR ABDOMINAL SURGERY
}

\author{
N. Salman', H. Ozay', U. Sabuncu', S. Yagar', B. Bostancı², S. Sekerci². \\ ISBU Turkiye Yuksek Ihtisas Ankara Training and Education Hospital, Department of Anesthesiology and Reanimation, Ankara, \\ TURKEY \\ ${ }^{2}$ SBU Turkiye Yuksek Ihtisas Ankara Training and Education Hospital, Department of Gastroenterological Surgery, Ankara,TURKEY
}

\section{Background and Goal of Study:}

Perioperative fluid and blood transfusion administrations are very important for hemodynamic stability, oxygen delivery and organ perfusion $(1,2)$. The aim of this study is to evaluate intraoperative fluid resuscitation in patients who underwent high risk major abdominal surgery and to compare the mortality and morbidity of intraoperative restrictive blood transfusion versus liberal blood transfusion.

\section{Materials and Methods:}

Among three hundred and fifty-two ASA (American Society of Anesthesiologists) scores were III-V patients who have had elective major abdominal surgery between July 20I4-July 2015,141 were included in the study. Patients were divided into two groups according to their blood transfusion management as "restrictive" (group R) and "liberal" (group L). Demographic data, preoperative, intraoperative, and postoperative complications, duration of hospitalization, intensive care unit stay, mortality and discharge rates were investigated.

\section{Results and Discussion:}

Demographic and preoperative data were similar in both groups (group L, $n=49$; and group $R, n=92$ ). However, the number of ASA IV patients in Group R was higher than Group $L(p<0.005)$. Intraoperative data (preoperative and postoperative haemoglobin values, type of surgery, duration of operation, total amount of crystalloid and colloid, fresh frozen plasma and erythrocyte suspension counts) were similar in both groups and it was seen that Group $L$ had more colloid transfusion than Group R. Postoperative complications, mortality/discharge ratio, duration of hospitalization and intensive care unit stay were similar in both groups. However, the rate of minor infections was $26.5 \%$ in Group $L$ and $7.6 \%$ in Group R, which was statistically significantly higher $(p=0.002)$.

\section{Conclusion(s):}

In high-risk patients, restrictive blood transfusion strategies should be used due to the positive effects of morbidity, mortality, and discharge compared to liberal blood transfusion strategies without applying fluid-restricting protocols.

Table 2. Intraoperative variables

\begin{tabular}{|c|c|c|c|}
\hline & $\begin{array}{r}\text { Grup L } \\
(n=49)\end{array}$ & $\begin{array}{c}\text { Grup R } \\
(\mathrm{n}=92)\end{array}$ & $p$ \\
\hline $\begin{array}{c}\text { Operation (\%) } \\
\text {-pancreas } \\
\text {-hepatic/biliary } \\
\text {-colorectal }\end{array}$ & $\begin{array}{l}36,7 \\
26,5 \\
36,7\end{array}$ & $\begin{array}{l}29,3 \\
27,2 \\
43,5\end{array}$ & 0.636 \\
\hline Preoperative $\mathrm{Hb}\left(\right.$ gr.dL $\left.^{-1}\right)($ mean $)$ & $12,3 \pm 1,9$ & $12,4 \pm 2,0$ & 0.585 \\
\hline Postoperative $\mathrm{Hb}\left({\mathrm{gr} . \mathrm{dL}^{-1}}^{-1}\right.$ (mean) & $11,6 \pm 1,7$ & $11,3 \pm 1,9$ & 0.303 \\
\hline $\begin{array}{l}\text { Intraoperative total crystalloid }(\mathrm{mL}) \\
\qquad(\text { mean })\end{array}$ & $2411,2 \pm 979,1$ & $2316,3 \pm 1285,9$ & 0.652 \\
\hline $\begin{array}{l}\text { Intraoperative total verilen kolloid }(\mathrm{mL}) \\
\text { (mean) }\end{array}$ & $515,3 \pm 474,3$ & $359 \pm 315,2$ & $0.021^{*}$ \\
\hline Intraoperative total FFP (Ü) (mean) & $0,6 \pm 1,2$ & $0,5 \pm 1,5$ & 0.447 \\
\hline Intraoperative total ES (Ü) (mean) & $0,6 \pm 0,9$ & $0,5 \pm 1,0$ & 0.588 \\
\hline Operation time (min) (mean) & $279,2 \pm 104,4$ & $307,2 \pm 159,1$ & 0.268 \\
\hline
\end{tabular}

\section{Table I. Demografic variables}

\begin{tabular}{|c|c|c|c|}
\hline & $\begin{array}{c}\text { Grup L } \\
(n=49)\end{array}$ & $\begin{array}{c}\text { Grup R } \\
(n=92)\end{array}$ & $p$ \\
\hline Age (mean) & $61,55 \pm 8,9$ & $63,45 \pm 9,6$ & 0.581 \\
\hline $\begin{array}{l}\text { Gender } \\
\text { M } \\
\text { F }\end{array}$ & $\begin{array}{l}63,3 \\
36,7\end{array}$ & $\begin{array}{l}69,6 \\
30,4\end{array}$ & 0.447 \\
\hline BMI (mean) & $27,7 \pm 5,3$ & $26,8 \pm 5,3$ & 0.377 \\
\hline $\begin{array}{c}\text { ASA }(\%) \\
\text { III } \\
\text { IV } \\
\text { V }\end{array}$ & $\begin{array}{c}100 \\
0 \\
0\end{array}$ & $\begin{array}{c}82,6 \\
17,4 \\
0\end{array}$ & $0.002^{*}$ \\
\hline $\begin{array}{c}\text { Smokers (\%) } \\
(+) \\
(-)\end{array}$ & $\begin{array}{l}67,3 \\
32,7\end{array}$ & $\begin{array}{l}65,2 \\
34,8\end{array}$ & 0.799 \\
\hline $\begin{array}{c}\text { Alcohol use }(\%) \\
(+) \\
(-)\end{array}$ & $\begin{array}{c}2 \\
98\end{array}$ & $\begin{array}{c}3,3 \\
96,7\end{array}$ & 0.678 \\
\hline $\begin{array}{c}\text { Preoperative cardiac disease (\%) } \\
(+) \\
(-)\end{array}$ & $\begin{array}{l}10,2 \\
89,8\end{array}$ & $\begin{array}{l}14,1 \\
85,9\end{array}$ & 0.506 \\
\hline $\begin{array}{c}\mathrm{DM} \\
(+) \\
(-)\end{array}$ & $\begin{array}{l}42,9 \\
53,1\end{array}$ & $\begin{array}{l}54,3 \\
45,7\end{array}$ & 0.194 \\
\hline $\begin{array}{l}\text { Preoperative neurological disorder } \\
\qquad \begin{array}{c}(\%) \\
(+) \\
(-)\end{array}\end{array}$ & $\begin{array}{c}4,1 \\
95,9\end{array}$ & $\begin{array}{c}4,3 \\
95,7\end{array}$ & 0.941 \\
\hline $\begin{array}{c}\mathrm{RF}(\%) \\
(+) \\
(-)\end{array}$ & $\begin{array}{c}0 \\
100\end{array}$ & $\begin{array}{c}3,3 \\
96,7\end{array}$ & 0.201 \\
\hline $\begin{array}{c}\text { COPD }(\%) \\
(+) \\
(-)\end{array}$ & $\begin{array}{l}53,1 \\
46,9\end{array}$ & $\begin{array}{l}38 \\
62\end{array}$ & 0.087 \\
\hline
\end{tabular}

Table 3. Postoperative variables

\begin{tabular}{|c|c|c|c|}
\hline & $\begin{array}{c}\text { Grup L } \\
(n=49)\end{array}$ & $\begin{array}{c}\text { Grup R } \\
(\mathrm{n}=92)\end{array}$ & $p$ \\
\hline $\begin{array}{c}\text { Minor infection (\%) } \\
(+) \\
(-)\end{array}$ & $\begin{array}{l}26,5 \\
73,5\end{array}$ & $\begin{array}{c}7,6 \\
92,4\end{array}$ & $0.002 *$ \\
\hline $\begin{array}{c}\text { Major infection (\%) } \\
(+) \\
(-)\end{array}$ & $\begin{array}{c}8,2 \\
91,8\end{array}$ & $\begin{array}{l}13 \\
87\end{array}$ & 0.384 \\
\hline $\begin{array}{c}\text { SVE }(\%) \\
(+) \\
(-)\end{array}$ & $\begin{array}{l}0 \\
0\end{array}$ & $\begin{array}{l}0 \\
0\end{array}$ & 1.000 \\
\hline $\begin{array}{c}\text { MI (\%) } \\
(+) \\
(-)\end{array}$ & $\begin{array}{c}0 \\
100\end{array}$ & $\begin{array}{c}1,1 \\
98,9\end{array}$ & 0.464 \\
\hline $\begin{array}{c}\text { DVT (\%) } \\
(+) \\
(-)\end{array}$ & $\begin{array}{l}0 \\
0\end{array}$ & $\begin{array}{l}0 \\
0\end{array}$ & 1.000 \\
\hline $\begin{array}{c}\text { PE (\%) } \\
(+) \\
(-)\end{array}$ & $\begin{array}{c}0 \\
100\end{array}$ & $\begin{array}{c}1,1 \\
98,9\end{array}$ & 0.464 \\
\hline $\begin{array}{c}\mathrm{DIC}(\%) \\
(+) \\
(-)\end{array}$ & $\begin{array}{l}0 \\
0\end{array}$ & $\begin{array}{l}0 \\
0\end{array}$ & 1.000 \\
\hline $\begin{array}{c}\mathrm{RF}(\%) \\
(+) \\
(-)\end{array}$ & $\begin{array}{c}0 \\
100\end{array}$ & $\begin{array}{c}1,1 \\
98,9\end{array}$ & 0.464 \\
\hline $\begin{array}{c}\text { Exitus (\%) } \\
\text { Discharged (\%) }\end{array}$ & $\begin{array}{c}6,1 \\
93,9\end{array}$ & $\begin{array}{c}4,8 \\
95,2\end{array}$ & 0.735 \\
\hline ICU time (day) (mean) & $5,9 \pm 9,6$ & $6,1 \pm 7,7$ & 0.804 \\
\hline Hospital stay time (day) (mean) & $11,4 \pm 7,3$ & $14,5 \pm 10,9$ & 0.074 \\
\hline
\end{tabular}

